\title{
A study of the origin of the electric activity of the rectum: Is it neurogenic or myogenic?
}

\author{
Ahmed Shafik \\ Professor and Chairman, Department of Surgery and Experimental Research, Faculty of Medicine, Cairo University, \\ Cairo, Egypt
}

The rectum possesses electric activity, the origin of which is yet undetermined. The current study investigates the possible source of these waves.

Three electrodes were sutured serially to the serosal surface of the rectum in 10 dogs. The rectal pressure was measured by a perfused catheter. Simultaneous recordings of the electric activity and rectal pressure were done before and after bilateral pelvic ganglionectomy and rectal myotomy.

Regular slow waves or pacesetter potentials (PPs) followed by inconsistent action potentials (APs) were recorded. They exhibited the same frequency, amplitude and velocity from three electrodes in the individual animal. APs were associated with minor rectal pressure rise. After pelvic ganglionectomy, PPs and APs were recorded but with irregular frequency, amplitude and conduction, a picture of 'rectoarrhythmia'. The rectoarrhythmic waves were registered proximally but not distally to the myotomy.

In conclusion, the rectal electric waves persist after bilateral pelvic ganglionectomy but exhibit a 'rectoarrhythmic' pattern. This is suggested to indicate that the waves are not initiated by, but may be under the control of, the extrarectal autonomic innervation. A 'pacemaker' is postulated to exist at the rectosigmoid junction triggering impulses that spread in the rectal wall along the muscle bundles or the myenteric nerve plexus.

Keywords: rectum; electrorectogram; slow waves; pacesetter potentials; action potentials; pelvic ganglia

\section{Introduction}

Recent studies have shown that the rectum possesses an electric activity which could be recorded in dogs and in humans by intrarectal as well as transcutaneous electrorectogram. ${ }^{1-3}$ Specific electrorectographic patterns were identified in various pathologic conditions of the rectum. ${ }^{4-6}$

The rectal electric activity manifested as slow waves or pacesetter potentials (PPs) and action potentials (APs). ${ }^{1-6}$ The PPs showed the same regular rhythm, frequency and amplitude when the test was repeated in the individual subject. PPs were followed by bursts of APs which had inconsistent frequency and were associated with a minor rectal pressure increase, indicating rectal motile activity. Studies have suggested that the PPs start at the rectosigmoid junction triggered by the rectosigmoid pacemaker, and propagate caudally. ${ }^{1}$ It was proposed that these waves are responsible for the motile activity of the rectum and that rectal electric activity disorders could lead to rectal dysmotility and defecation disorders. ${ }^{1-6}$ However, the exact source of these electric waves is as yet not ascertained. They are suggested to originate from the rectal wall which

Correspondence: A Shafik, 2 Talaat Harb Street, Cairo, Egypt contains neural and myogenic elements. Consequently, they could arise from any of these structures.

The purpose of this communication is to study the potential origin of the rectal electric waves. As they play a significant role in the motile activity of the rectum, the elucidation of their origin might assist in furthering our understanding of rectal motility and its disorders. The study was approved by our Faculty Review Board.

\section{Material and methods}

\section{Material}

The study comprised 10 mongrel dogs (seven male, three female) with a mean weight of $14.7+2.6$ SD kg (range 12-17). The animals were kept in cages 1 week prior to the study to get accustomed to the facilities. They were treated in accordance with the 'Guide for the Care and Use of Laboratory Animals'?

\section{Methods}

The dogs were premedicated with acepromazine $(0.15 \mathrm{mg} / \mathrm{kg})$ injected subcutaneously. They were 
anesthetized with intravenous sodium pentobarbital $(35 \mathrm{mg} / \mathrm{kg}$ ) given as a bolus injection at $20-25 \mathrm{mg} / \mathrm{h}$ to maintain adequate anesthesia with spontaneous respiration. All dogs were intubated to assist ventilation.

The abdomen was opened through a midline abdominal incision and the rectum was exposed. The electric activity of the rectum was recorded by means of monopolar silver-silver chloride electrodes. Each electrode had a diameter of $0.8 \mathrm{~mm}$ and was covered by an insulating vinyl sheath sparing its tip (Smith Kline Beckman, Los Angeles, California). Three electrodes were sutured serially to the serosal surface of the rectum (Figure 1) with a distance of approximately $1.5-2 \mathrm{~cm}$ between one electrode and the other. A metal cannula containing 3-pin sockets to which the electrodes were attached, was sutured to the anterior abdominal wall. The dog was awakened from anesthesia and given 2 weeks post-operatively for the wound to heal before the recordings were started.

Prior to the tests the animals were kept fasting for $12 \mathrm{~h}$ and a saline enema was performed. We waited for $1 \mathrm{~h}$ after enema administration so that its possible effect on the rectum would have disappeared and the recording was done. The insulated wire leads were attached to the pins in the cannula and connected to a Brush Mark 200 rectilinear pen recorder. The electric activity including the frequency, amplitude and velocity of conduction of the waves, was recorded from the three electrodes in each dog for periods of 30 min on 15 different days.

Simultaneous recording of the electrical and mechanical activity of the rectum was performed. The rectal pressure was measured by means of a $10-\mathrm{F}$ catheter with multiple side ports and a distal closed end. The catheter was placed in the rectum $4-5 \mathrm{~cm}$ from the anal oriface and was connected to a pneumohydraulic capillary infusion system (Arndorfer Medical Specialities, Greendale, Wisconsin, USA) which delivered saline continually via the capillary tube at a rate of $0.6 \mathrm{ml} / \mathrm{min}$. The transducer outputs were registered on a rectilinear recorder (RS 3400 Gould Inc, Ohio, USA). Occlusion of the recording orifice produced a rate of rising pressure greater than $250 \mathrm{~cm} \mathrm{H}_{2} \mathrm{O} / \mathrm{s}$.

\section{Rectal denervation}

Before producing rectal denervation, the response of the rectal pressure to an intravenous injection of $1 \mathrm{mg}$ of bethanechol chloride (urecholine) and $5 \mathrm{mg}$ of atropine, respectively, was registered. After the 15 days of electromechanical recording, the abdomen of the animals was reopened through the same incision, the right and left pelvic ganglia were exposed and bilateral ganglionectomy was performed which paralysed not only the rectum but also the urinary bladder. ${ }^{8}$ Therefore, cystostomy for urine drainage was done at the same setting.

Two weeks were allowed postoperatively for the wounds to heal. The urecholine and atropine tests were repeated to confirm rectal denervation. The electromechanical activity of the rectum was then recorded daily for 15 days as aforementioned.

\section{Rectal myotomy}

To determine the role of the rectal musculature in initiating electric activity, rectal myotomy was performed. The dogs were re-operated in the same way as aforementioned, and the rectum was exposed. An

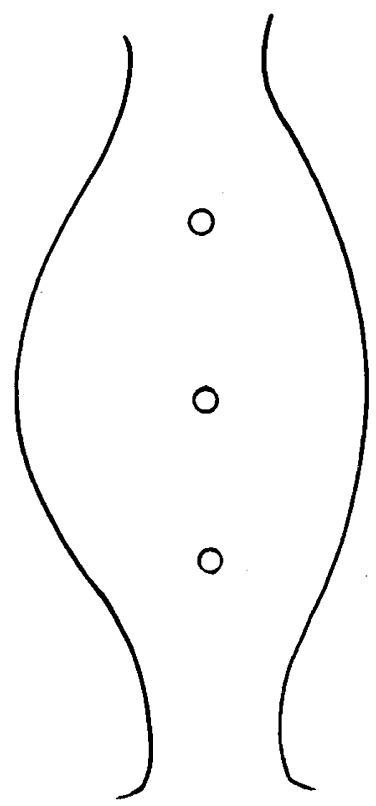

Figure 1 Sites of rectal electrodes

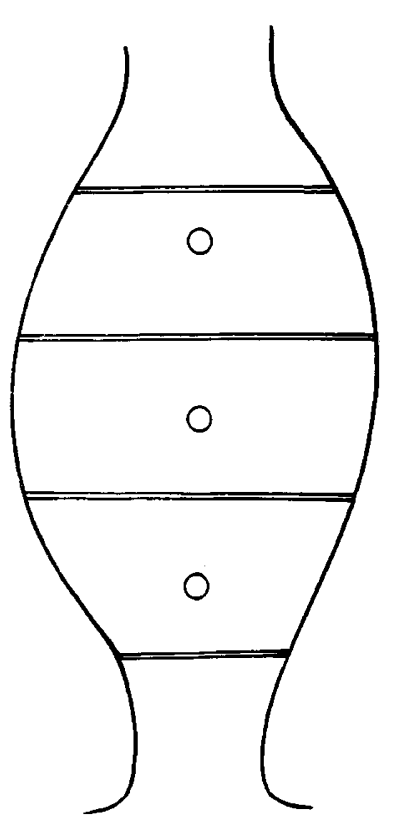

Figure 2 Sites of myotomies 
annular myotomy was performed in the rectal wall $1 \mathrm{~cm}$ above the upper electrode in two dogs, between electrodes 1 and 2 in two dogs, 2 and 3 in two dogs and distal to electrode 3 in another two dogs (Figure 2 ). The myotomy comprised annular division of the longitudinal and circular muscle coats of the rectum down to, but not including, the mucosa which bulged between the cut edges of the muscle. Two weeks postoperatively, the electromechanical activity was recorded as mentioned above.

Reproducibility was ascertained by repeating the recordings of the electromechanical activity more than twice in the same animal. The results were analyzed statistically using the Student's $t$-test. Differences assumed statistical significance at $P<0.05$, and values were given as mean \pm SD.

\section{Results}

No adverse effects occurred to the animals during or after performing the operations except for superficial wound infection in some of the dogs which healed under antibiotic cover. There was no electrode break or migration, and the electrodes were found in their sites each time abdominal exploration was carried out. The tests were completed and evaluated in all the dogs.
Slow waves or pacesetter potentials (PPs) were recorded from the three electrodes of each animal. The waves had a triphasic configuration which consisted of a small positive, a large negative and another small positive deflection (Figure 3 ). The shape was constant in all the recordings from the same site. The PPs exhibited the same frequency, amplitude and velocity of conduction by the three electrodes (Figure $3)$. The mean frequency was $5.1 \pm 1.6$ cycle per min (cpm; range 4-7), amplitude $0.58 \pm 0.08 \mathrm{mV}$ (range $0.4-0.75)$ and velocity of conduction $4.9 \pm 1.2 \mathrm{~cm} / \mathrm{s}$ (range $3.2-6.5$ ).

\section{Electromechanical activity}

Bursts of fast-activity spikes or action potentials (APs) were recorded. They took the form of negative deflections which followed the PPs randomly (Figure 3 ). They had the same pattern from the three electrodes of the individual animal. In contrast to the PPs, the APs were inconsistent when the test was repeated in the same dog.

The rectal pressure increased synchronously with the occurrence of APs (Figure 4). The mean rectal pressure at rest was $9.6 \pm 1.8 \mathrm{~cm} \mathrm{H}_{2} \mathrm{O}$ (range 8-11). During bursts of APs, it increased to $20.6 \pm 4.2 \mathrm{~cm}$ $\mathrm{H}_{2} \mathrm{O}$ (range $16-24$ ). The rectal pressure did not show

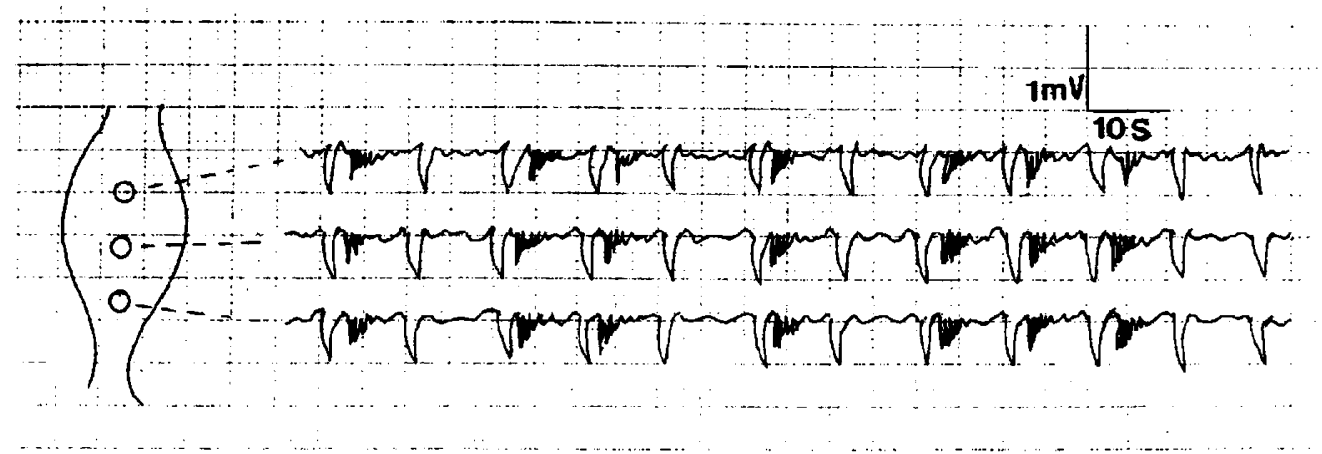

Figure 3 Electrorectogram showing the pacesetter and action potentials which have the same frequency, amplitude and velocity of conduction by the three electrodes

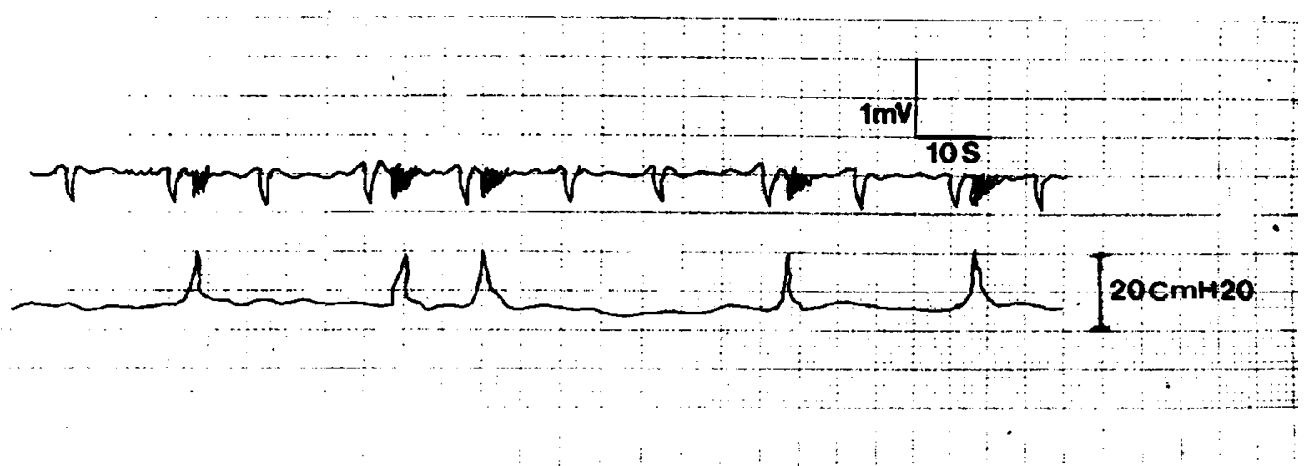

Figure 4 Electrorectogram (upper tracing) and rectal pressure (lower tracing) showing pressure rise with APs, and not with PPs 


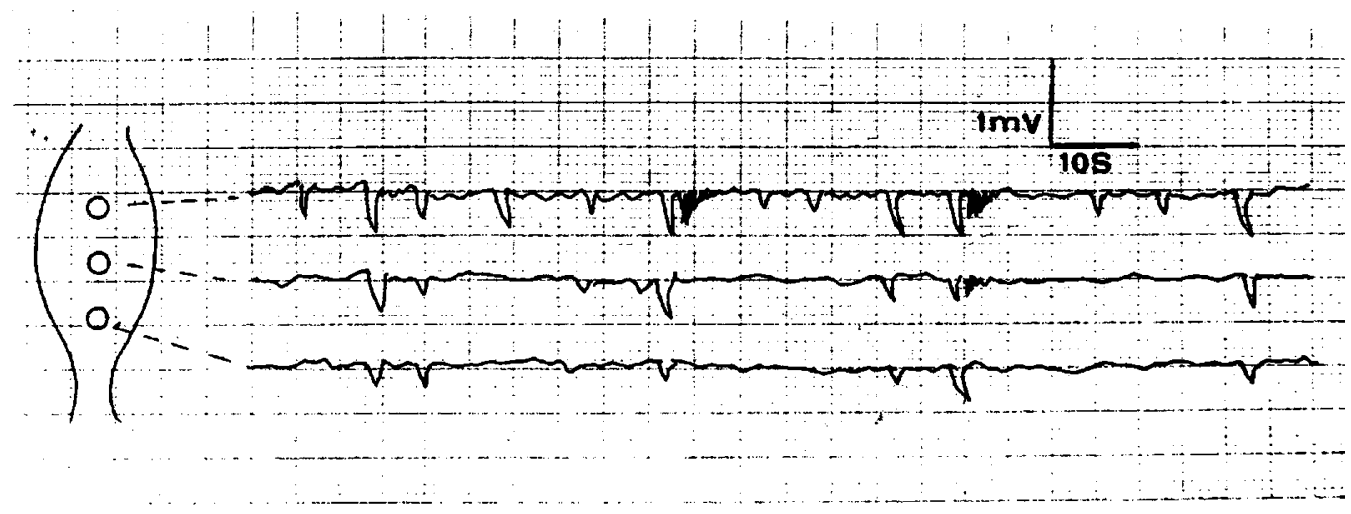

Figure 5 Electrorectogram after bilateral pelvic ganglionectomy showing the PPs with irregular frequency, amplitude and velocity of conduction from the three electrodes, a picture of 'rectoarrhythmia'

significant changes $(P>0.05)$ with the PPs. Due to the inconsistent occurrence of the APs, the rectal pressure rise was also inconsistent at test repetition in the individual dog on all test days.

Effect of rectal denervation on the rectal electric activity The mean resting rectal pressure was $9.6 \pm 1.8 \mathrm{~cm} \mathrm{H}_{2} \mathrm{O}$ (range 8-11). After a 1-mg urecholine injection, it rose to a mean of $31.6 \pm 7.2 \mathrm{~cm} \mathrm{H}_{2} \mathrm{O}$ (range 23-37, $P<0.01)$, and after $5 \mathrm{mg}$ atropine injection, it dropped to $2.9 \pm 1.1 \mathrm{~cm} \mathrm{H}_{2} \mathrm{O}$ (range $2-4, P<0.05$ ). After bilateral pelvic ganglionectomy, the resting rectal pressure dropped to a mean of $3.9 \pm 1.2 \mathrm{~cm} \mathrm{H}_{2} \mathrm{O}$ (range $2.5-5 ; \quad P<0.05$ ) and no significant rectal pressure response occurred to either injection of $1 \mathrm{mg}$ urecholine or $5 \mathrm{mg}$ atropine $(P>0.05)$.

Recordings of the rectal electromechanical activity after bilateral pelvic ganglionectomy revealed that PPs and APs were still registered. However, they showed irregular frequency, amplitude and velocity of conduction from the same electrode. These variables were not identical in the three electrodes of the individual animal (Figure 5); furthermore, they differed from one day to the other. A picture of 'rectoarrhythmia' was recorded from the electrodes in all of the dogs.

\section{Effect of rectal myotomy on electromechanical activity of the rectum}

After rectal myotomy cranial to electrode 1 , the three electrodes did not show electric activity (Figure 6a). When the myotomy was performed between electrodes 1 and 2, electric activity could be recorded from electrode 1, but not from electrodes 2 and 3 (Figure 6b). Myotomy between electrodes 2 and 3 showed the electric activity from electrodes 1 and 2 but not from electrode 3 (Figure 6c). The electric waves were recorded from the three electrodes when the myotomy was performed distal to them (Figure 6d). The above recorded electric activity showed a picture of rectoar- rhythmia. No retrograde or abnormal waves were recorded by any of the electrodes.

The aforementioned results were reproducible with no significant difference $(P>0.05)$ when the tests were repeated in the same animal.

\section{Discussion}

Electroenterography is becoming an investigative tool in the diagnosis of gastro-intestinal disorders. It shows changes in pathologic conditions of the stomach, ${ }^{9-11}$ colon $^{12-15}$ and rectum ${ }^{3-6}$ especially in motility disorders. The main components of the electric activity are the slow waves which are superimposed or followed by bursts of spike activity or action potentials. The former are regular while the latter occur randomly. The action potentials are associated with increased intraluminar pressure and are likely to play a role in the motile activity of the gut. $1,15-17$

The current study has shown that electric activity could be recorded from the rectum as regular and reproducible PPs with inconsistent APs. After bilateral pelvic ganglionectomy, the electric signals could still be recorded but with irregular frequency, amplitude and rhythm, a condition we call 'rectoarrhythmia'. This suggests that the electric waves are initiated in the rectal wall and not produced by the extrarectal autonomic innervation. Nevertheless, the irregular rhythm of the waves after bilateral pelvic ganglionectomy seems to denote that these waves, though not being produced by, are under the control of the autonomic innervation.

In the rectal wall, the electric waves appear to originate from either the enteric plexus of nerves or the muscle fibers. Previous studies ${ }^{1}$ have demonstrated that the electric waves spread caudad and this could be confirmed in the current study. When the rectal musculature was interrupted by myotomies, the electric signals showed proximal but not distal to the cut. These results are suggested to indicate that the waves are initiated in the proximal part of the rectum and spread caudally and that they are not created 

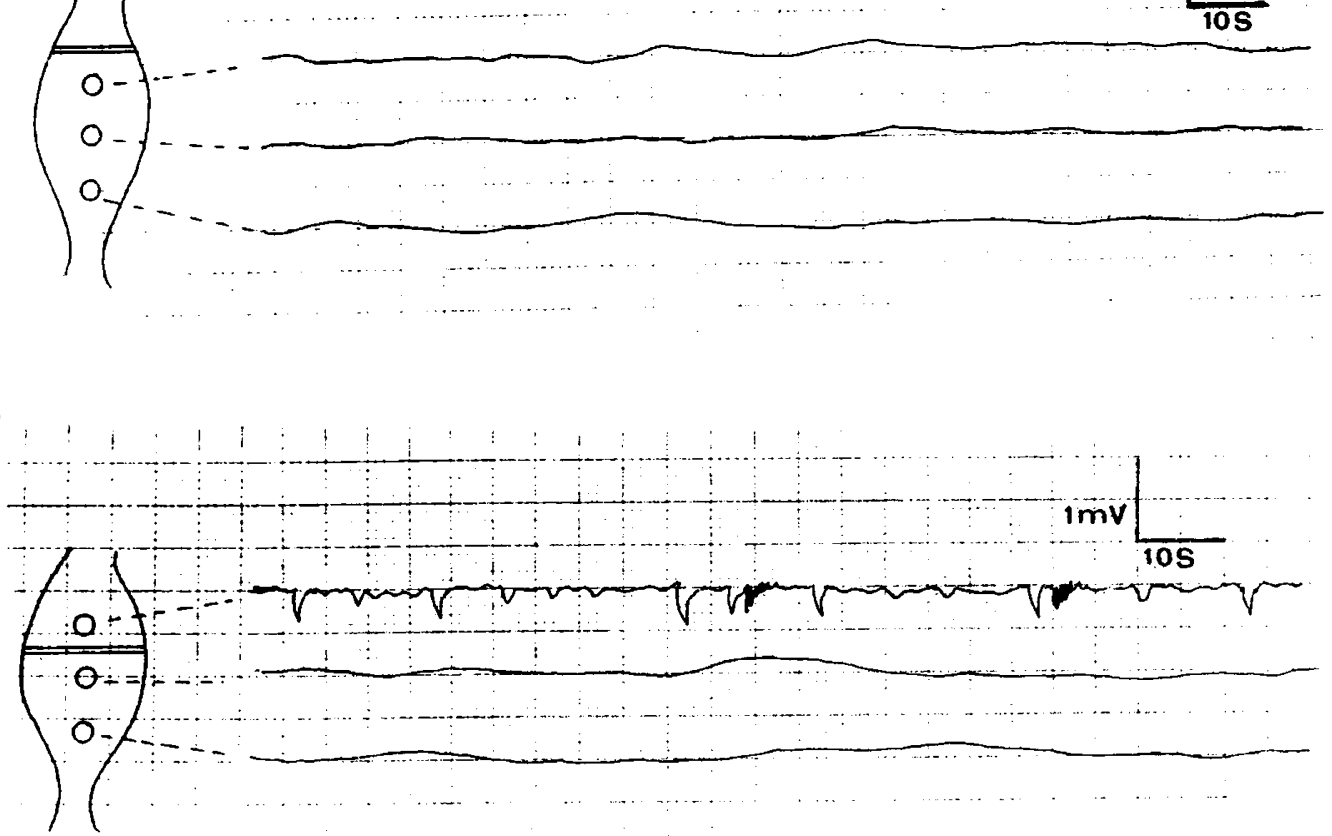

c

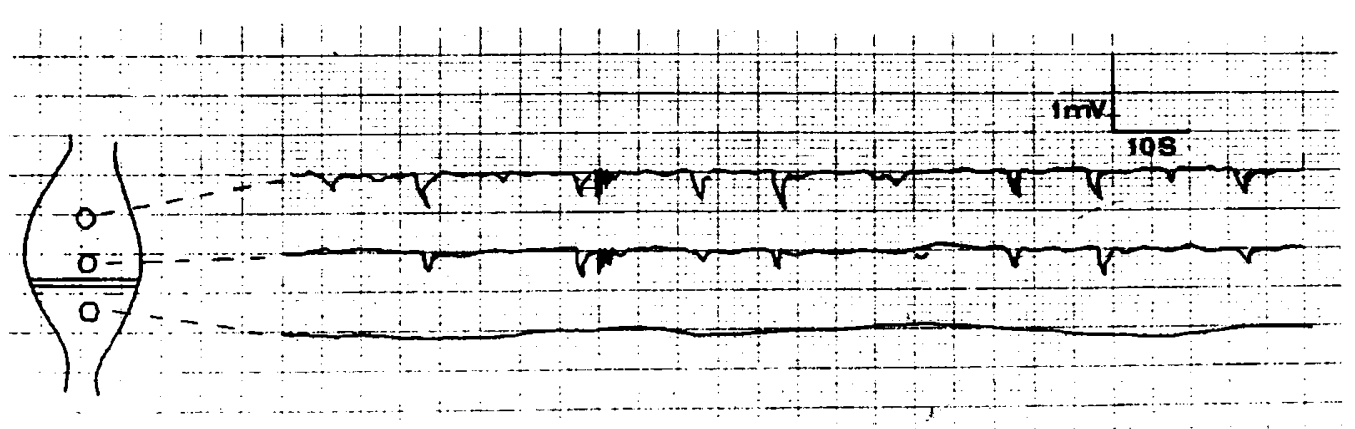

d

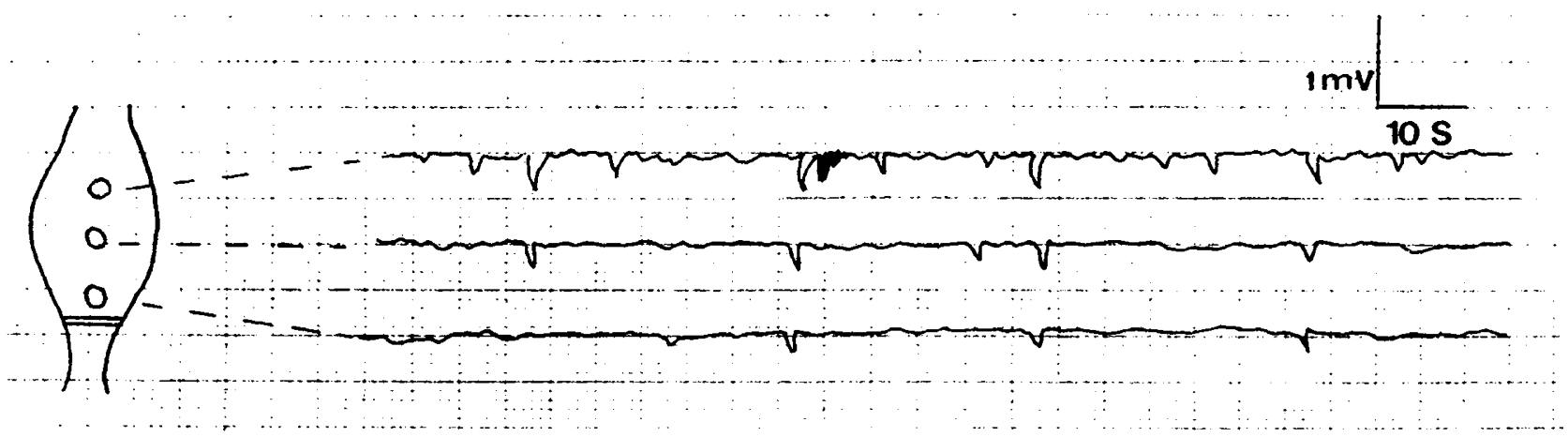

Figure 6 Rectal myotomy (a) distal to the three electrodes, (b) between electrodes 1 and 2, (c) 2 and 3, and (d) distal to the three electrodes 
segmentally. It seems that they are evoked by a 'pacemaker' which appears to exist in the upper part of the rectum at the rectosigmoid junction. According to our earlier study ${ }^{1}$ a myotomy proximal to the rectosigmoid junction did not affect the initiation or conduction of the PPs in the rectal wall.

\section{A concept of origin and transmission of the electric waves in the rectal wall}

Considering the findings of the current and previous studies, ${ }^{1}$ we postulate that a 'pacemaker' exists in the upper rectum triggering impulses which seem to spread in the rectal wall along the myenteric plexus of nerves. A neurotransmitter is probably secreted at the neuromuscular junction. Alternatively, the action potentials beginning in the pacemaker might spread directly to rectal muscle bundles. As the rectal musculature is syncythial ${ }^{18}$ the wave spreads along the entire rectal muscle mass.

It is furthermore speculated that these waves are not to be considered a continuation of those of the sigmoid colon. A previous study ${ }^{19}$ has shown that the rectal electric waves differ from those of the sigmoid with regard to the frequency, amplitude, and velocity of conduction indicating that the sigmoid and rectal electric activity have probably two different sources.

The concept of the caudad spread of the rectal electric waves originating from a pacemaker located in the upper part of the rectum might explain the 'mass squeeze' contraction of rectal detrusor. ${ }^{20}$ Mass squeezing comprises sequential continuous rectal contraction starting at the rectosigmoid junction and spreading caudad in the rectum, compressing its contents in one direction. This 'mass squeeze' motile activity is probably induced by the electric waves which seem to be initiated by the pacemaker.

The neuropathic rectum that occurs in spinal cord injuries and other neurologic conditions, is associated with defecation disorders. These disorders are believed to be due to the irregular electric activity (rectoarrhythmia) with a resulting disturbed motility. Further studies are indicated to confirm this hypothesis.

In conclusion, the aforementioned findings suggest that the electric waves are initiated in the rectal wall and in the same time are under the control of the autonomic innervation. Furthermore, it is speculated that defecation disorders occurring in the neuropathic rectum are due to the disturbed rectal electric activity.

\section{Acknowledgements}

Waltraut Reichelt and Margot Yehia assisted in preparing the manuscript.

\section{References}

1 Shafik A. Study of the electrical and mechanical activity of the rectum. Experimental study. Eur Surg Res 1994; 26: 87-93.

2 Shafik A. Study of the electromechanical activity of the rectum. Human study. Coloproctology 1993; 15: 215-218.

3 Shafik A, Nour A, Abdel-Fattah A. Transcutaneous electrorectography. Human electrorectogram from surface electrodes. Digestion 1995: 56: $479-482$.

4 Shafik A. Electrorectography in chronic constipation. World J Surg 1995: 19: $772-775$.

5 Shafik A. Electrorectogram in the neuropathic rectum. Paraplegia 1995: 33: $346-349$.

6 Shafik A. Electrorectogram in Hirschsprung's disease. Ped Surg Int 1995: 10: $478-480$.

7 The Guide for Care and Use of Laboratory Animals. US Public Health Services NIH Pub No. 1: 5-23, 1989.

8 Shafik A. Reinnervation of the rectum with a somatic nerve: a canine study. Spinal Cord 1997; 35: 53-57.

9 Geldof H, Van der Schee EJ, Van Blankenstein M, Grashuis JL. Electrogastrographic study of gastric myoelectrical activity in patients with unexplained nausea and vomiting. Gut 1986; 27: $799-808$.

10 Abell TL, Malagelada JR. Glucagon-evoked gastric dysrhythmias in humans shown by an improved electrogastrographic technique. Gastroenterology 1985: 88: 1932-1940.

11 Pezzolla F, Piezzo G, Maselli MA, Giorgio I. Gastric electrical dysrhythmias following cholecystectomy in humans. Digestion 49: $134-139$.

12 Schang JC, Devroede G. Fasting and postprandial myoelectric spiking in the human sigmoid colon. Gastroenterology 1983; 85: $1048-1053$.

13 Dapoigny M, Trolese JF, Bommerlae G, Tournut R. Myoelectric spiking activity of right colon, left colon and rectosigmoid of healthy humans. Dig Dis Sci 1988; 33: 1007-1012.

14 Frexinos J, Bueno L, Fuiramonti J. Diurnal changes in myoelectric spiking activity of the human colon. Gastroenterology 1985; 88: $1104-1110$.

15 Shafik A. Transcutaneous electrosigmoidography. Study of the myoelectric activity of sigmoid colon by surface electrodes. Front Biosci 1996; 1: b1 - 4, PubMed No: 9159196.

16 Garcia D, et al. Colonic motility: electric and manometric description of mass movement. Dis Colon Rectum 1991; 34: 577 584.

17 Bassotti G, Morelli A, Whitehead WE. Abnormal rectosigmoid myoelectric response to eating in patients with severe idiopathic constipation (slow transit type). Dis Colon Rectum 1992; 35: $753-756$.

18 Junqueira LC, Carniero J, Long JA. Muscle tissue. In: Junqueira LC, Carniero J, Long JA (eds) Basic Histology: 5th edition, Los Altos: Lange Medical Publications, 1986; pp 234-254.

19 Shafik A. A study of the functional activity of the sigmoid colon and rectum during fecal storage in the sigmoid. Gut 1996; Suppl 3, 39: A 264 No 1584 (abstr).

20 Shafik A, Moneim KA. Dynamic study of the rectal detrusor activity at defecation. Digestion 1991; 49: $167-174$. 